\title{
Relationship of postpartum interval to estrus, body condition score, milk yield and blood biochemical parameters in Surti buffaloes (Bubalus bubalis)
}

\author{
P.M. Gamit ${ }^{1}$, Rana Ranjeet Singh ${ }^{2 *}$, Amit Kumar ${ }^{3}$, V.B. Kharadi ${ }^{4}$ and N.B. Patel ${ }^{5}$ \\ ${ }^{1}$ Cattle breeding farm, JAU, Junagadh, Gujarat, INDIA \\ ${ }^{2 *}$ Department of LPM, VCVS\&AH, NAU, Navsari, Gujarat, INDIA \\ ${ }^{3}$ LAR section, AG division, IVRI, Bareilly, Uttar Pradesh, INDIA \\ ${ }^{4}$ LRS, NAU, Gujarat, INDIA \\ ${ }^{5}$ Department of LPM, VCVS\&AH, NAU, Navsari, Gujarat, INDIA \\ *Corresponding author. E-mail: drexplicit@gmail.com
}

Received: October 15, 2015; Revised received: March 1, 2016; Accepted: May 30, 2016

\begin{abstract}
The aim of the present investigation was to find out the relationship among postpartum interval to estrus, body condition score, milk yield and blood biochemical parameters of Surti buffaloes (Bubalus bubalis). The study was conducted on sixteen clinically healthy Surti buffaloes (parity 1-7) with normal parturition. These animals were divided into two groups on the basis of their postpartum interval to estrus (PPIE). Group 1 animals had PPIE $\leq 50$ days whereas group 2 had PPIE > 50 days. Body condition score (BCS), milk yield and Blood samples were collected by jugular venipuncture on days starting from $6^{\text {th }}$ day after calving thereafter at fortnight interval till $90^{\text {th }}$ day postpartum. Blood serum parameters such as glucose, total protein, blood urea, creatinine, cholesterol, triglyceride, progesterone and estrogen were measured. Perusal of data revealed that animals having higher BCS on the day of estrus had significantly $(P \leq 0.05)$ shorter PPIE. There was non-significant effect of daily and cumulative 100 days milk yield on PPIE. Serum concentration of glucose and creatinine was significantly $(P \leq 0.05)$ higher for group 1 animals at most of the stages. There was non-significant difference between serum concentration of total protein, blood urea nitrogen and cholesterol between both the groups. Progesterone and Estradiol-17 $\beta$ concentrations were significantly $(P \leq 0.05)$ higher in group 1 animals than group 2 animals at different stages of this study.
\end{abstract}

Keywords: Blood-biochemical profile, Body condition score, Hormonal profile, Postpartum interval to estrus, Surti buffaloes

\section{INTRODUCTION}

India is having highest number of buffaloes $(105.3$ million) in the world and more than half (54.47 million) of this population is adult female buffalo. Surti is small-medium size, well-defined milch breed of buffalo, well adapted to the climate of Central and South Gujarat region and are mainly kept by the landless, small and marginal farmers of this area (Kharadi et al., 2006). The goal of reproduction management is to have cows become pregnant at a biologically optimal time and at an economically profitable interval after calving (Zaleha et al., 2013). Body condition score, milk yield and various blood metabolites are known to affect resumption of postpartum cyclicity in buffaloes (Tripathi et al., 2010; Mohan et al., 2010; Khan et al., 2011; Ali and Shukla, 2012; Banu et al., 2012; Jayachandran et al., 2013). Metabolic changes in blood affect biochemical composition of the follicular fluid and indirectly influence oocyte quality (Ali et al., 2008, Abd Ellah, 2010). In the bovines, expression of oestrus and ovulation is the result of highly synchronized hormonal milieu during the periestrual period
(Layek et al., 2013). Milk/plasma progesterone and/ oestradiol-17 $\beta$ concentration is a good predictor to determine the ovarian follicular dynamics and functional status of the corpus luteum in bovines (Presicce et al., 2005, Honparkhe et al., 2008). Information on female sexual behaviour of buffaloes is few and in India no structured information is available for these traits for Surti buffaloes under this agro-climatic region. Therefore, present investigation was undertaken with the objective to study the relationship among postpartum interval to estrus, body condition score, milk yield and blood biochemical parameters of Surti buffaloes (Bubalus bubalis).

\section{MATERIALS AND METHODS}

Present investigation was conducted on sixteen Surti buffaloes which calved from October 2013 to February 2014 at Livestock Research Station, Navsari Agricultural University, Navsari, Gujarat, India. All the buffaloes under study had normal calving and subsequent good genital health as assessed by rectal palpation. The animals were maintained under loose housing and group management system. The housing space for the 
animals was specified as per BIS .Animal shed had both covered and open paddock. All management practices were uniform to all animals. They were allowed to feed in continuous feeding manager inside the shed and were also allowed for grazing for 4-5 hours during day time. The animals had free access to fresh and clean drinking water all the time. Evidence of female sexual activities of Surti buffaloes were observed after calving till the animal shows the signs of heat or $90^{\text {th }}$ day postpartum. Animals were exposed to teaser bull for 20-30 minutes three times a day i.e. 6:00 AM, 12:00 noon and 6:00 PM, to identify the cows in heat. Rectal palpation of uterine tone and presence of follicle on the surface of the ovary was done during these periods. Number of days after calving, animal shows the signs of first heat was observed and recorded. Body condition score, milk yield and blood samples were collected from animals on $6^{\text {th }}, 20^{\text {th }}, 34^{\text {th }}, 48^{\text {th }} 62^{\text {nd }} 76^{\text {th }}$, $90^{\text {th }}$ day postpartum and on the day of estrus. Body condition score of animals were recorded as per the body condition scoring chart formulated by Alapati et al. (2010). Milk yield (morning and evening) of individual buffalo was recorded by electronic weighing balance. Blood samples $(10 \mathrm{ml})$ were collected by puncturing the jugular vein of the individual animals into the vacutainers. Serum was separated by centrifugation at $3000 \mathrm{rpm}$ for 15 minutes and stored at $-20{ }^{\circ} \mathrm{C}$ in deepfreeze until analyzed. Each of these samples were analyzed for serum concentration of glucose (enzymatic GOD-PAP method), total protein (Biuret method), total cholesterol (Enzymatic Endpoint method), triglycerides (GPO-PAP method), blood urea (enzyme kinetic method), creatinine (Liquid) using assay kit and Merck single beam spectrophotometer (Thermo Fischer Scientific Inc, Madison, USA). Serum progesterone $\left(\mathrm{P}_{4}\right)$ and Oestradiol $\left(\mathrm{E}_{2}\right)$ concentration was measured by standard Enzyme Linked Immuno Sorbent Assay (ELISA) technique using assay kit. Obtained data were classified according to number of days after which animals showed first sign of heat postpartum. Group 1 animal which shoed first heat on or before 50 days postpartum and group 2 included animals which showed signs of heat after 50 days postpartum. The collected data has been compiled, tabulated and analyzed by using PROC GLM procedure of SAS 9.3.

\section{RESULTS AND DISCUSSION}

The PPIE for group 1 animals was $38.14 \pm 2.36$ days while it was $58.67 \pm 3.55$ days for group 2 animals. Body condition score of group 1 animals were slightly higher than the group 2 animals at all stage however this difference was non-significant except on the day of estrus where it was significantly $(\mathrm{P} \leq 0.05)$ higher for group 1 animals than the group 2 animals (Table 1). Further, we observed that the BCS showed a decreasing trend up to $76^{\text {th }}$ day postpartum in group 1 animals while in group 2 animals it was either static or decreasing till stage $90^{\text {th }}$ day postpartum. Our findings are in agreement with the findings of Ruegg et al. (1992), Hady et al. (1994), Hegazy et al. (1997), Baruselli et al. (2001), Kadarmideen and Wegmann (2003), Anitha

Table 1. Least squares' means and standard error $(\mathrm{LSM} \pm \mathrm{SE})$ of $\mathrm{BCS}$ as per PPIE groups at different stages.

\begin{tabular}{lllllllll}
\hline & \multicolumn{7}{c}{ Days (postpartum) } \\
\cline { 2 - 8 } $\begin{array}{l}\text { PPIE } \\
\text { groups }\end{array}$ & $\mathbf{6}^{\text {th }}$ & $\mathbf{2 0}^{\text {th }}$ & $\mathbf{3 4}^{\text {th }}$ & $\mathbf{4 8}^{\text {th }}$ & $\mathbf{6 2}^{\text {nd }}$ & $\mathbf{7 6}^{\text {th }}$ & $\mathbf{9 0}^{\text {th }}$ & $\begin{array}{l}\text { On the } \\
\text { day of } \\
\text { estrus }\end{array}$ \\
\hline $\begin{array}{l}1(38.14 \pm 2.36 \\
\text { days) }\end{array}$ & $3.08 \pm 0.10(9)$ & $2.91 \pm 0.13(9)$ & $2.88 \pm 0.13(9)$ & $2.89 \pm 0.14(9)$ & $2.80 \pm 0.14(9)$ & $2.76 \pm 0.12(9)$ & $2.79 \pm 0.09(9)$ & $2.87 \pm 0.11^{\mathrm{a}}(9)$ \\
$\begin{array}{l}2(58.67 \pm 3.55 \\
\text { days) }\end{array}$ & $2.90 \pm 0.07(7)$ & $2.68 \pm 0.09(7)$ & $2.67 \pm 0.09(7)$ & $2.68 \pm 0.10(7)$ & $2.69 \pm 0.07(7)$ & $2.66 \pm 0.08(7)$ & $2.56 \pm 0.09(7)$ & $2.57 \pm 0.08^{\mathrm{b}}(7)$ \\
OVERALL & $2.98 \pm 0.06(16)$ & $2.78 \pm 0.08(16)$ & $2.77 \pm 0.08(16)$ & $2.77 \pm 0.09(16)$ & $2.74 \pm 0.07(16)$ & $2.70 \pm 0.07(16)$ & $2.66 \pm 0.07(16)$ & $2.70 \pm 0.07(16)$ \\
\hline
\end{tabular}

LSM showing different superscripts in lower case letters in a column differ significantly $(\mathrm{P} \leq 0.05)$; Figures in parentheses show the number of animal to derive LSM

Table 2. Least squares' means and standard error $(\mathrm{LSM} \pm \mathrm{SE})$ of cumulative milk yield (in 100 days) and daily milk yield (kg) as per PPIE groups at different stages.

\begin{tabular}{llllllllll}
\hline & & \multicolumn{7}{c}{ Daily milk yield (in Kg) at different stages } \\
\cline { 3 - 9 } $\begin{array}{l}\text { PYIE } \\
\text { groups }\end{array}$ & $\begin{array}{l}\text { MY100 } \\
\mathbf{k g}\end{array}$ & $\mathbf{6}^{\text {th }}$ & $\mathbf{2 0}^{\text {th }}$ & $\mathbf{3 4}^{\text {th }}$ & $\mathbf{4 8}^{\text {th }}$ & $\mathbf{6 2}^{\text {nd }}$ & $\mathbf{7 6}^{\text {th }}$ & $\mathbf{9 0}^{\text {th }}$ & $\begin{array}{l}\text { On the } \\
\text { day of } \\
\text { estrus }\end{array}$ \\
\hline $1(38.14 \pm$ & $555.34 \pm 21.59$ & $3.24 \pm 0.32$ & $4.99 \pm 0.40$ & $5.21 \pm 0.43$ & $5.61 \pm 0.48$ & $5.83 \pm 0.32$ & $5.77 \pm 0.56$ & $5.91 \pm 0.23$ & $5.70 \pm 0.36$ \\
2.36 days) & $(9)$ & $(9)$ & $(9)$ & $(9)$ & $(9)$ & $(9)$ & $(9)$ & $(9)$ & $(9)$ \\
$2(58.67 \pm$ & $529.47 \pm 42.09$ & $2.89 \pm 0.28$ & $4.67 \pm 0.40$ & $4.86 \pm 0.39$ & $5.04 \pm 0.45$ & $5.23 \pm 0.34$ & $5.53 \pm 0.33$ & $5.47 \pm 0.39$ & $5.31 \pm 0.34$ \\
3.55 days) & $(7)$ & $(7)$ & $(7)$ & $(7)$ & $(7)$ & $(7)$ & $(7)$ & $(7)$ & $(7)$ \\
OVER- & $540.79 \pm 24.98$ & $3.04 \pm 0.21$ & $4.81 \pm 0.28$ & $5.01 \pm 0.28$ & $5.29 \pm 0.33$ & $5.49 \pm 0.24$ & $5.64 \pm 0.30$ & $5.66 \pm 0.25$ & $5.48 \pm 0.25$ \\
ALL & $(16)$ & $16)$ & $(16)$ & $(16)$ & $(16)$ & $(16)$ & $(16)$ & $(16)$ & $(16)$ \\
\hline
\end{tabular}

Figures in parentheses show the number of animals to derive LSM 
Table 3. Least squares' means and standard error $(\mathrm{LSM} \pm \mathrm{SE})$ of serum biochemical parameters as per PPIE groups at different stages.

\begin{tabular}{|c|c|c|c|c|c|c|c|c|c|}
\hline \multirow[b]{2}{*}{ PARAMETER } & \multirow{2}{*}{$\begin{array}{l}\text { GROUP } \\
1(n=7) \& 2 \\
(n=9)\end{array}$} & \multicolumn{8}{|c|}{ STAGES } \\
\hline & & $6^{\text {th }}$ & $20^{\text {th }}$ & $34^{\text {th }}$ & $48^{\text {th }}$ & $62^{\text {nd }}$ & $76^{\text {th }}$ & $90^{\text {th }}$ & DAY OF ESTRUS \\
\hline \multirow{4}{*}{ Glucose (mg/dl) } & 1 & $\begin{array}{l}58.16 \pm 1 . \\
05^{\mathrm{a}}\end{array}$ & $\begin{array}{l}58.84 \pm 0.8 \\
6\end{array}$ & $\begin{array}{l}60.25 \pm 0 . \\
79^{\mathrm{a}}\end{array}$ & $\begin{array}{l}61.78 \pm 0 \\
.90\end{array}$ & $\begin{array}{l}63.87 \pm \\
0.75^{\mathrm{a}}\end{array}$ & $\begin{array}{l}64.52 \pm \\
0.78\end{array}$ & $\begin{array}{l}65.33 \pm \\
0.92^{\mathrm{a}}\end{array}$ & $59.63 \pm 1.67$ \\
\hline & 2 & $\begin{array}{l}54.26 \pm 1 \\
04^{\mathrm{b}}\end{array}$ & $\begin{array}{l}56.01 \pm 1.0 \\
1\end{array}$ & $\begin{array}{l}57.89 \pm 0 . \\
70^{\mathrm{b}}\end{array}$ & $\begin{array}{l}59.89 \pm 0 \\
.92\end{array}$ & $\begin{array}{l}61.33 \pm \\
0.84^{\mathrm{b}}\end{array}$ & $\begin{array}{l}64.30 \pm \\
0.78\end{array}$ & $\begin{array}{l}62.64 \pm \\
0.82^{\mathrm{b}}\end{array}$ & $56.18 \pm 1.67$ \\
\hline & OVER ALL & $\begin{array}{l}55.97 \pm 0 \\
04\end{array}$ & $\begin{array}{l}57.25 \pm 0.7 \\
5\end{array}$ & $\begin{array}{l}58.92 \pm 0 \\
59\end{array}$ & $\begin{array}{l}60.77 \pm 0 \\
.67\end{array}$ & $\begin{array}{l}62.44 \pm \\
0.64\end{array}$ & $\begin{array}{l}64.40 \pm \\
1.22\end{array}$ & $\begin{array}{l}63.82 \pm \\
0.68\end{array}$ & $57.69 \pm 0.98$ \\
\hline & 1 & $\begin{array}{l}7.72 \pm 0.1 \\
7\end{array}$ & $7.86 \pm 0.19$ & $\begin{array}{l}8.04 \pm 0.2 \\
0\end{array}$ & $\begin{array}{l}8.15 \pm 0 . \\
18\end{array}$ & $\begin{array}{l}8.28 \pm 0 . \\
19\end{array}$ & $\begin{array}{l}8.43 \pm 0 . \\
18\end{array}$ & $\begin{array}{l}8.54 \pm 0 . \\
17\end{array}$ & $8.54 \pm 0.19$ \\
\hline \multirow{2}{*}{$\begin{array}{l}\text { Total protein } \\
(\mathrm{g} / \mathrm{dl})\end{array}$} & 2 & $\begin{array}{l}7.19 \pm 0.2 \\
1\end{array}$ & $7.44 \pm 0.19$ & $\begin{array}{l}7.65 \pm 0.1 \\
6\end{array}$ & $\begin{array}{l}7.76 \pm 0 . \\
16\end{array}$ & $\begin{array}{l}7.89 \pm 0 . \\
16\end{array}$ & $\begin{array}{l}7.94 \pm 0 . \\
16\end{array}$ & $\begin{array}{l}8.13 \pm 0 . \\
17\end{array}$ & $8.15 \pm 0.19$ \\
\hline & OVER ALL & $\begin{array}{l}7.42 \pm 0.1 \\
5\end{array}$ & $7.63 \pm 0.14$ & $\begin{array}{l}7.82 \pm 0.1 \\
3\end{array}$ & $\begin{array}{l}7.93 \pm 0 . \\
13\end{array}$ & $\begin{array}{l}8.06 \pm 0 . \\
13\end{array}$ & $\begin{array}{l}8.16 \pm 0 . \\
13\end{array}$ & $\begin{array}{l}8.31 \pm 0 . \\
13\end{array}$ & $8.32 \pm 0.14$ \\
\hline \multirow{4}{*}{$\begin{array}{l}\text { Blood urea } \\
\text { concentration } \\
(\mathrm{mg} / \mathrm{dl})\end{array}$} & 1 & $\begin{array}{l}39.94 \pm 1 \\
51\end{array}$ & $\begin{array}{l}50.88 \pm 0.4 \\
6\end{array}$ & $\begin{array}{l}51.21 \pm 1 \\
07\end{array}$ & $\begin{array}{l}49.26 \pm 0 \\
.94\end{array}$ & $\begin{array}{l}56.15 \pm \\
1.20^{\mathrm{a}}\end{array}$ & $\begin{array}{l}51.49 \pm \\
0.90\end{array}$ & $\begin{array}{l}55.11 \pm \\
1.06\end{array}$ & $51.15 \pm 0.71$ \\
\hline & 2 & $\begin{array}{l}36.47 \pm 0 \\
97\end{array}$ & $\begin{array}{l}50.91 \pm 0.7 \\
9\end{array}$ & $\begin{array}{l}48.97 \pm 0 . \\
82\end{array}$ & $\begin{array}{l}48.13 \pm 0 \\
.70\end{array}$ & $\begin{array}{l}53.09 \pm \\
0.79^{\mathrm{b}}\end{array}$ & $\begin{array}{l}53.36 \pm \\
0.54\end{array}$ & $\begin{array}{l}54.37 \pm \\
0.77\end{array}$ & $50.47 \pm 0.53$ \\
\hline & OVER ALL & $\begin{array}{l}37.99 \pm 0 . \\
94\end{array}$ & $\begin{array}{l}50.90 \pm 0.4 \\
8\end{array}$ & $\begin{array}{l}49.95 \pm 0 \\
70\end{array}$ & $\begin{array}{l}48.63 \pm 0 \\
.57\end{array}$ & $\begin{array}{l}54.43 \pm \\
0.77\end{array}$ & $\begin{array}{l}52.54 \pm \\
0.53\end{array}$ & $\begin{array}{l}54.69 \pm \\
0.62\end{array}$ & $51.67 \pm 0.44$ \\
\hline & 1 & $\begin{array}{l}0.97 \pm 0.0 \\
4^{\mathrm{a}}\end{array}$ & ${ }_{\mathrm{a}}^{1.13 \pm 0.06}$ & $\begin{array}{l}1.22 \pm 0.0 \\
6^{\mathrm{a}}\end{array}$ & $\begin{array}{l}1.29 \pm 0 . \\
06^{\mathrm{a}}\end{array}$ & $\begin{array}{l}1.36 \pm 0 . \\
05^{\mathrm{a}}\end{array}$ & $\begin{array}{l}1.41 \pm 0 . \\
05\end{array}$ & $\begin{array}{l}1.51 \pm 0 . \\
04\end{array}$ & $1.58 \pm 0.03$ \\
\hline \multirow{2}{*}{$\begin{array}{l}\text { Creatinine } \\
(\mathrm{mg} / \mathrm{dl})\end{array}$} & 2 & $\begin{array}{l}0.81 \pm 0.0 \\
6^{\mathrm{b}}\end{array}$ & $\begin{array}{l}0.89 \pm 0.06 \\
\mathrm{~b}\end{array}$ & $\begin{array}{l}0.97 \pm 0.0 \\
7^{\mathrm{b}}\end{array}$ & $\begin{array}{l}1.06 \pm 0 . \\
07^{\mathrm{b}}\end{array}$ & $\begin{array}{l}1.15 \pm 0 . \\
07^{\mathrm{b}}\end{array}$ & $\begin{array}{l}1.25 \pm 0 . \\
07\end{array}$ & $\begin{array}{l}1.39 \pm 0 . \\
06\end{array}$ & $1.45 \pm 0.05$ \\
\hline & OVER ALL & $\begin{array}{l}0.88 \pm 0.0 \\
4\end{array}$ & $1.00 \pm 0.05$ & $\begin{array}{l}1.08 \pm 0.0 \\
6\end{array}$ & $\begin{array}{l}1.16 \pm 0 . \\
05\end{array}$ & $\begin{array}{l}1.24 \pm 0 . \\
05\end{array}$ & $\begin{array}{l}1.32 \pm 0 . \\
05\end{array}$ & $\begin{array}{l}1.44 \pm 0 . \\
04\end{array}$ & $1.51 \pm 0.04$ \\
\hline \multirow{4}{*}{$\begin{array}{l}\text { Cholesterol } \\
\text { concentration } \\
(\mathrm{mg} / \mathrm{dl})\end{array}$} & 1 & $\begin{array}{l}99.56 \pm 4 \\
62\end{array}$ & $\begin{array}{l}108.22 \pm 4 \\
79\end{array}$ & $\begin{array}{l}116.10 \pm \\
4.04\end{array}$ & $\begin{array}{l}119.48 \pm \\
4.42\end{array}$ & $\begin{array}{l}126.61 \\
\pm 2.86\end{array}$ & $\begin{array}{l}129.54 \\
\pm 2.91\end{array}$ & $\begin{array}{l}132.68 \\
\pm 2.91\end{array}$ & $123.31 \pm 1.40$ \\
\hline & 2 & $\begin{array}{l}92.17 \pm 4 \\
62\end{array}$ & $\begin{array}{l}100.52 \pm 3 \\
96\end{array}$ & $\begin{array}{l}106.79 \pm \\
3.50\end{array}$ & $\begin{array}{l}112.88 \pm \\
3.07\end{array}$ & $\begin{array}{l}122.27 \\
\pm 2.77\end{array}$ & $\begin{array}{l}126.86 \\
\pm 2.15\end{array}$ & $\begin{array}{l}130.55 \\
\pm 2.09\end{array}$ & $119.36 \pm 1.64$ \\
\hline & OVER ALL & $\begin{array}{l}95.40 \pm 3 \\
00\end{array}$ & $\begin{array}{l}103.89 \pm 3 \\
11\end{array}$ & $\begin{array}{l}110.87 \pm \\
2.82\end{array}$ & $\begin{array}{l}115.77 \pm \\
2.64\end{array}$ & $\begin{array}{l}124.17 \\
\pm 2.01\end{array}$ & $\begin{array}{l}128.03 \\
\pm 1.73\end{array}$ & $\begin{array}{l}131.48 \\
\pm 1.55\end{array}$ & $121.08 \pm 1.19$ \\
\hline & 1 & $\begin{array}{l}44.22 \pm 1 \\
28\end{array}$ & $\begin{array}{l}42.18 \pm 1.1 \\
2\end{array}$ & $\begin{array}{l}42.88 \pm 1 \\
12\end{array}$ & $\begin{array}{l}47.47 \pm 1 \\
.40\end{array}$ & $\begin{array}{l}49.38 \pm \\
1.60\end{array}$ & $\begin{array}{l}50.90 \pm \\
1.67\end{array}$ & $\begin{array}{l}48.03 \pm \\
1.60\end{array}$ & $51.74 \pm 0.62$ \\
\hline \multirow{2}{*}{$\begin{array}{l}\text { Triglyceride } \\
\text { concentration } \\
(\mathrm{mg} / \mathrm{dl})\end{array}$} & 2 & $\begin{array}{l}43.33 \pm 1 \\
13\end{array}$ & $\begin{array}{l}40.17 \pm 1.3 \\
5\end{array}$ & $\begin{array}{l}40.65 \pm 1 \\
57\end{array}$ & $\begin{array}{l}43.48 \pm 1 \\
.64\end{array}$ & $\begin{array}{l}45.85 \pm \\
1.54\end{array}$ & $\begin{array}{l}48.44 \pm \\
1.51\end{array}$ & $\begin{array}{l}49.69 \pm \\
1.19\end{array}$ & $53.80 \pm 0.69$ \\
\hline & OVER ALL & $\begin{array}{l}43.72 \pm 0 . \\
82\end{array}$ & $\begin{array}{l}41.05 \pm 0.9 \\
1\end{array}$ & $\begin{array}{l}41.63 \pm 1 \\
02\end{array}$ & $\begin{array}{l}45.23 \pm 1 \\
.19\end{array}$ & $\begin{array}{l}47.39 \pm \\
1.17\end{array}$ & $\begin{array}{l}49.52 \pm \\
1.13\end{array}$ & $\begin{array}{l}48.96 \pm \\
0.96\end{array}$ & $52.89 \pm 0.53$ \\
\hline
\end{tabular}

LSM showing different superscripts in lower case letters in a column differ significantly $(\mathrm{P} \leq 0.05)$; Figures in parentheses show the number of animal to derive LSM

Table 4. Least squares' means and standard error $(\mathrm{LSM} \pm \mathrm{SE})$ of serum hormonal parameters as per PPIE groups at different stages.

\begin{tabular}{|c|c|c|c|c|c|c|c|c|c|}
\hline \multirow{2}{*}{$\begin{array}{l}\text { PARAME- } \\
\text { TER }\end{array}$} & \multirow{2}{*}{$\begin{array}{l}\text { GROUP } \\
1(n=7) \& \\
2(n=9)\end{array}$} & \multicolumn{8}{|c|}{ STAGES } \\
\hline & & $6^{\text {th }}$ & $20^{\text {th }}$ & $34^{\text {th }}$ & $48^{\text {th }}$ & $62^{\text {nd }}$ & $76^{\text {th }}$ & $90^{\text {th }}$ & $\begin{array}{l}\text { DAY OF } \\
\text { ESTRUS }\end{array}$ \\
\hline \multirow{3}{*}{$\begin{array}{l}\text { Progesterone } \\
\text { (P4) } \\
\text { concentration } \\
(\mathrm{ng} / \mathrm{ml})\end{array}$} & 1 & $0.32 \pm 0.11$ & $1.21 \pm 0.17^{\mathrm{a}}$ & $2.06 \pm 0.31^{\mathrm{a}}$ & $1.58 \pm 0.30$ & $1.70 \pm 0.37$ & $2.05 \pm 0.26$ & $1.81 \pm 0.28$ & $0.67 \pm 0.03$ \\
\hline & 2 & $0.27 \pm 0.13$ & $0.69 \pm 0.13^{b}$ & $1.19 \pm 0.25^{b}$ & $1.87 \pm 0.26$ & $2.07 \pm 0.25$ & $2.05 \pm 0.25$ & $1.51 \pm 0.26$ & $0.59 \pm 0.03$ \\
\hline & OVER ALL & $0.29 \pm 0.06$ & $0.92 \pm 0.12$ & $1.57 \pm 0.22$ & $1.74 \pm 0.19$ & $1.91 \pm 0.21$ & $2.05 \pm 0.18$ & $1.64 \pm 0.17$ & $0.63 \pm 0.02$ \\
\hline \multirow{3}{*}{$\begin{array}{l}\text { Estrogen } \\
\text { (E2) } \\
\text { concentration } \\
(\mathrm{pg} / \mathrm{ml})\end{array}$} & 1 & $42.99 \pm 0.99$ & $18.99 \pm 1.27$ & $16.15 \pm 0.99$ & $17.97 \pm 0.72^{\mathrm{a}}$ & $21.40 \pm 1.09$ & $18.69 \pm 0.66$ & $19.29 \pm 1.5$ & $54.82 \pm 1.97$ \\
\hline & 2 & $40.73 \pm 1.18$ & $17.66 \pm 2.23$ & $15.21 \pm 0.97$ & $16.08 \pm 0.53^{\mathrm{b}}$ & $18.75 \pm 0.98$ & $17.20 \pm 0.51$ & $17.97 \pm 1.45$ & $53.85 \pm 1.35$ \\
\hline & OVER ALL & $41.72 \pm 0.82$ & $18.24 \pm 1.34$ & $15.62 \pm 0.69$ & $16.91 \pm 0.48$ & $19.91 \pm 0.78$ & $17.85 \pm 0.44$ & $18.55 \pm 1.04$ & $54.28 \pm 1.11$ \\
\hline
\end{tabular}

LSM showing different superscripts in lower case letters in a column differ significantly $(\mathrm{P} \leq 0.05)$. Figures in parentheses show the number of animal to derive LSM 
et al. (2011) and Banu et al. (2012) where they reported that buffaloes those had higher body condition were found to have shorter postpartum anoestrus interval than those with lower body condition score. Further, Buckley et al. (2003) reported that the likelihood of reproductive success was best predicted by BCS around the time of first service. There was nonsignificant difference in the milk yield (100 days) as well as in daily milk yield between both the groups though it was higher for group 1 animals than the group 2 animals (Table 2). Land and Leaver (1981), Van Eerdenburg et al. (2002), Banu et al. (2012) and Hussein et al. (2013) also reported non-significant influence milk production on the interval to first estrus in buffaloes. Serum glucose concentrations $(\mathrm{mg} / \mathrm{dl})$ of group 2 animals were significantly $(\mathrm{P} \leq 0.05)$ lower than the group 1 animals on $6^{\text {th }}, 34^{\text {th }}, 62^{\text {nd }}$ and $90^{\text {th }}$ day postpartum, respectively. Further, we observed that the glucose concentration showed increasing trend from $6^{\text {th }}$ day to $90^{\text {th }}$ day postpartum for group 1 animal and up to $76^{\text {th }}$ day postpartum for group 2 animals (Table 3 ). This might be due to the fact that cows in moderate to thin condition had greater requirement for glucose than cows in a good condition due to increased maintenance requirements and therefore increased glucose utilization and reduced serum glucose concentration (Adams et al., 1987). Findings of this study are in agreement with the findings of Reist et al. (2002), who reported a negative correlation with energy balance and concentration of glucose $(\mathrm{r}=-0.457)$ in high yielding dairy cows. Total protein concentrations $(\mathrm{g} / \mathrm{dl})$ were within the normal physiological range and in group 1 animal it was slightly higher than the group 2 animals during entire study period however this difference was statistically non-significant. Further, total protein concentration showed increasing trend from $6^{\text {th }}$ day to 90 day postpartum for both the groups. Similar findings were reported by Yaylak et al. (2009), Aktas et al. (2011) and Dubey (2013), who observed low serum total protein concentration at early stages of lactation and in the course of time its concentrations increased. Blood urea concentration $(\mathrm{mg} / \mathrm{dl})$ was almost within the normal physiological range but there was no definite trend of serum blood urea values in both the groups. The blood urea concentration $(\mathrm{mg} / \mathrm{dl})$ of group 1 animals were significantly $(\mathrm{P} \leq 0.05)$ higher than the group 2 animals at 62 day postpartum only. Creatinine concentrations $(\mathrm{mg} / \mathrm{dl})$ of group 1 animals were significantly $(\mathrm{P} \leq 0.05)$ higher than the group 2 animals between $6^{\text {th }}$ day to $62^{\text {nd }}$ day postpartum, respectively. Further, we found that the creatinine concentration was lowest at $6^{\text {th }}$ day postpartum for both group of animals while highest values were observed on the day of estrus (Table 3). Comparable values of creatinine was reported by Ghuman et al. (2011) in cyclic and non-cyclic buffaloes. Total cholesterol concentration showed increasing trend from $6^{\text {th }}$ to $90^{\text {th }}$ day postpartum in both the groups. The total cholesterol concentration for group 1 animals was higher than the group 2 animals during entire study period though this difference was statistically nonsignificant. Above findings might be due to the fact that cholesterol may be involved in steroid synthesis in ovary and luteal function hence might have influenced PPIE. Similar findings were reported by Reist et al. (2002) who found that the cholesterol were positively associated with energy balance, reflecting metabolic adaptation after parturition. There was non-significant difference in the triglyceride concentration in both groups of animals. However, triglyceride concentrations $(\mathrm{mg} / \mathrm{dl})$ of group 1 animals were higher than the group 2 animals during entire study period except on the day of estrus. Above findings might be due to the fact that dairy cows mobilizes body reserve mainly fat reserves and produce glycerol for energy resources and consequently NEFA concentration increases. The released NEFA offers 3 metabolic interests firstly, they are utilised for milk fat synthesis by the mammary gland, secondly these may constitute an energy source for peripheral tissues and finally these may be esterified into triglycerides by the liver exported throughout VLDL formation. Similar, findings were reported by Yaylak (2009), Aktas et al. (2011) and Dubey (2013), they observed that thin cow due to insufficient lipomobilisation capacity (BCS $\leq$ 2.50 units), the FFA, triglyceride and VLDL concentrations may be low than cows with medium BCS exhibited significantly increased NEFA proportions, and increased serum and triglyceride concentrations. Progesterone concentrations $(\mathrm{ng} / \mathrm{ml})$ were significantly $(\mathrm{P} \leq 0.05)$ higher in group 1 animals than group 2 animals at stage $20^{\text {th }}$ and $34^{\text {th }}$ day postpartum. During $46^{\text {th }}$ to $76^{\text {th }}$ day postpartum its values was lower in group 2 than group 1. Koenen and Veerkamp (1999) and Lucy (2001) reported that low BCS or higher loss of BCS may influence reproduction by delaying first ovulation postpartum by reducing progesterone production in cyclic cattle which is in agreement with our findings. Estradiol-17 $\beta$ concentrations ( $\mathrm{pg} / \mathrm{ml})$ were significantly $(\mathrm{P} \leq 0.05)$ higher in group 1 animals than group 2 animals at stage $48^{\text {th }}$ day postpartum (Table 4 ). We found that the concentration of the estrogen was highest on day of estrus in both groups. In our study, it has been observed that the animals having comparatively higher BCS came to heat earlier and had comparatively higher level of estradiol-17 $\beta$ than the animals with lesser BCS. Our findings are in accordance with the findings of Malfatti (2003) and Dubey (2013) who observed that animals with higher BCS had higher level of estradiol-17 $\beta$ than the animals with lesser BCS.

\section{Conclusion}

Animals having higher BCS on the day of estrus had significantly shorter PPIE. There was non-significant effect of daily and cumulative 100 days milk yield on PPIE. Serum concentration of glucose and creatinine was significantly $(\mathrm{P} \leq 0.05)$ higher for group 1 animals 
at most of the stages. Progesterone and Estradiol-17 $\beta$ concentrations were significantly $(\mathrm{P} \leq 0.05)$ higher in group 1 animals than group 2 animals at different stages of this study.

\section{REFERENCES}

Abd Ellah, M.R. (2010). Serum biochemical reference values for female buffaloes in Egypt. Buffalo Bulletin, 29:141147.

Adams, D.C., Short, R.E. and Khapp, B.W. (1987). Body size and body condition effects on performance and behavior grazing beef cows. Nutrition Reports International, 35: 269-277.Aktas, M.S., Ozkanlar, S., Ucar, O., Ozkanlar, Y., Kaynar, O. and Aytekin, I. (2011). Relationships between body condition score and some metabolic blood parameters in early lactating dairy cows. Revue de Médecine Vétérinaire, 162 (12): 586-592.

Alapati, A., Rao, S. K., Suresh, J., Srinivasa, M.P.R. and Kotilinga, R. (2010). Development of the body condition score system in Murrah buffaloes: validation through ultrasonic assessment of body fat reserves. Journal of Veterinary Science, 11: 1-8.

Ali, R. and Shukla, S.P. (2012). Haemato-biochemical changes in post-partum anoestrus buffaloes during low breeding season. Researcher, 4:55-58.

Ali, S., Ahmad, N., Akhtar, N., Zia-ur-Rahman. and Noakes, D.E. (2008). Metabolite contents of blood serum and fluid from small and large sized follicles in dromedary camels during the peak and the low breeding seasons. Animal Reproduction Science, 108: 446-456.

Anitha, A., Rao, K.S., Suresh, Moorthy, J.P.R.S. and Reddy, Y.K. (2011). A body condition score (BCS) system in Murrah buffaloes. Buffalo Bulletin, 30: 79-99.

Banu, T.A., Shamsuddin, M., Bhattacharjee, J., Islam, M.F., Khan, S.I. and Ahmed, J.U. (2012). Milk progesterone enzyme-linked immunosorbent assay as a tool to investigate ovarian cyclicity of water buffaloes in relation to body condition score and milk production. Acta Veterinaria Scandinavica, 54:30.

Baruselli, P.S., Barnabe, V.H.., Barnabe, R.C.., Visintin, J.A.., Molero-Filho, J.R. and Porto, R.(2001). Effect of body condition score at calving on postpartum reproductive performance in buffaloes. Buffalo Journal, 1: 53 -65 .

Buckley, F., Sullivan, K.O., Mee, J.F., Evans, R.D. and Dillon, P. (2003). Relationship among milk yield, body condition, cow weight and reproduction in springcalved Holstein Friesians. Journal of Dairy Science, 86: 2308-2319.

Dubey, P. (2013). Study on dam-calf interaction and postpartum oestrus behaviour in Surti buffaloes. M.V.Sc. Thesis submitted to Navsari Agricultural University, Navsari, Gujarat.

Ghuman, S.P.S., Singh, J., Honparkhe, M., Ahuja, C.S., Dhami, D.S., Nazir, G. and Gandotra, V.K. (2011). Differential fertility in dairy buffalo: Role of thyroid and blood plasma biochemical milieu. Iranian Journal of Appllied Animal Science, 1: 105-109.

Hady, P.J., Domecq, J.J. and Kaneene, J.B. (1994). Frequency and precisions of body condition scoring in dairy cattle. Journal of Dairy Science, 77: 1543-1547.

Hegazy, M.A., Essawi, S.A. and Youssef, A.H. (1997). Relationship between body condition, milk yield, and reproductive performance. Veterinary Medical Journal, Giza,
45: 147-154.

Honparkhe, M., Singh, J., Dadarwal, D., Dhaliwal, G.S. and Kumar, A. (2008). Estrus induction and fertility rates in response to exogenous hormonal administration in postpartum anestrous and subestrous bovines and buffaloes. Journal of Veterinary Medical Science, 70:1327-1331.

Hussein, H. A., Waleed, S. and Mahmoud, R. A. (2013). Relationship among uterine involution, ovarian activity, blood metabolites and subsequent reproductive performance in Egyptian buffaloes. Open Journal of Animal Sciences, 3: 59-69.

Jayachandran, S., Nanjappan, K., Muralidharan, J., Selvaraj, P. and Manoharan, A. (2013). Blood biochemical and mineral status in cyclic and postpartum anestrus buffaloes. International Journal of Food, Agriculture and Veterinary Sciences, 3: 93-97.

Kadarmideen, H.N. and Wegmann, S. (2003). Genetic relationship among body condition score, type, and fertility and production traits in Swiss Holstein cattle 50 years of DNA. In: Proceedings of the Fifteenth Conference, Association for the Advancement of Animal Breeding and Genetics, Melbourne. Austrailia, pp.77-81.

Khan, H.M., Mohanty, T.K., Bhakat, M., Raina, V.S. and. Gupta, A.K. (2011). Relationship of Blood Metabolites with Reproductive Parameters during Various Seasons in Murrah Buffaloes. Asian-Australian. Journal of Animal Science, 24: 1192-1198.

Kharadi, V.B., Desai, P.M., Sabapara, G.P., Vij, P.K., Singh, P.K., Singh, G. and Ahlawat, S.P.S. (2006). Buffalo geneteic resource of India-Surti. Monogram of ICAR Network project on Survey and Characterization of Surti buffalo breed in Gujarat published by National bureau of animal genetic resources, Karnal \& Navsari agricultural university, Navsari.

Koenen, E.P.C. and Veerkamp, R.F. (1999). Genetic covariance functions for live weight, condition score and dry matter intake measured at different lactation stages of Holstein Friesian heifers. Livestock Production Science, 57: 67-77.

Land, C. and Leaver, J.D. (1981). The effect of body condition at calving on the production of Friesian cows and heifers. Animal Production, 32: 362 (Abstr.).

Layek, S.S., Mohanty T.K., Kumaresan, A., Behera, K. and Chand, S. (2013). Cervical mucus characteristics and periestrual hormone concentration in relation to ovulation time in Zebu (Sahiwal) cattle. Livestock Science, 152: 273-281.

Lucy, M.C., Staples, C.R., Michel, F.M. and Thatcher, W.W. (1991). Energy balance and size and number of ovarian follicles detected by ultrasonography in early postpartum dairy cows. Journal of Dairy Science, 74: 473-482.

Malfatti, A. (2003). Recent advances in buffalo endocrinology. Atti Secondo Congresso Nazionale sull'Allevamento del Bufalo. Monterotondo, Roma, 28-30: 161176.

Mohan, K., Kumar, V., Sarkar, M. and Prakash, B.S. (2010). Temporal changes in endogenous estrogens and expression of behaviors associated with estrus during the periovulaory period in Murrah buffaloes (Bubalus bubalis). Tropical-Animal-Health-and-Production, 42: 21-26.

Presicce, G.A., Bella, A., Terzano, G.M., De Santis, G. and Santore, E.M. (2005). Postpartum ovarian follicular dynamics in primiparous and pluiriparous Mediterranean Italian buffaloes (Bubalus bubalis). Theriogenol- 
ogy, 63:1430-1439.

Reist, M., Erdin, D., VonEuw, D. and Tschuemperlin, K. (2002). Estimation of energy balance at the individual and herd level using blood and milk traits in high yielding dairy cows. Journal of Dairy Science, 85: 314-3327.

Ruegg, P.L., W.J. Goodger, C.A.Holmberg, L. D.Weaver, and E.M. Huffman. (1992). Relation among body condition score, milk production, and serum urea nitrogen and cholesterol concentrations in high-producing Holstein dairy cows in early lactation. American Journal of Veterinary Research, 53:5.

Tripathi, P.M., Ingole, S.D., Deshmukh, B.T., Nagvekar, A.S and Bharucha, S.V. (2010). Serum lipid profile during lactation in buffalo. Indian Journal of Animal Science,
80: 217-219.

Van Eerdenburg, F.J.C.M., Karthaus, D., Taverne, M.A.M., Merics, I. and Szenci, O. (2002). The relationship between estrous behavioural score and time of ovulation in dairy cattle. Journal of Dairy Science, 85:1150-1156.

Yaylak, E., Yenisey, C. and Seyrek, K. (2009). Effects of lameness, stage of lactation and body condition score on some blood parameters in Holstein cows. Asian Journal of Animal and Veterinary Advances, 4: 245-251.

Zaleha, P., Vargova, M., Kadasi, M., Smitka, P., Smarzik, V. and Kovae, G. (2013). Effect of post partum uterine involution on folliculogenesis, oestrus and conception in cows. Roczniki Naukowe Polskiego Towarzystwa Zootechnicznego, 9: 57-65. 